\title{
¿Por qué siguen siendo tan altas las tasas de mortalidad por malnutrición grave?'
}

\author{
C. Schofield ${ }^{2}$ y A. Ashworth ${ }^{2}$
}

RESUMEN La revisión de las publicaciones que han aparecido en los últimos cinco decenios revela que la mediana de la tasa de letalidad de la malnutrición grave no se ha modificado en ese período y que suele oscilar de 20 a 30\%, con los mayores porcentajes (50 a 60\%) atribuibles a los casos de malnutrición edematosa.

Una causa probable de la persistencia de la mortalidad elevada es el tratamiento inapropiado de los casos. Según una encuesta de centros de tratamiento en distintas partes del mundo $(\mathrm{n}=79)$, es común que a los niños con enfermedad aguda se les prescriban dietas inadecuadas con un alto contenido de proteínas, energía y sodio y un bajo contenido de micronutrientes. Se encontró que ciertas prácticas que podrían acarrear consecuencias funestas, como la prescripción de diuréticos para el edema, estaban ampliamente difundidas. También se comprobó que muchos de los manuales de instrucción eran anticuados y encerraban contradicciones. Ya que se pueden lograr tasas bajas de mortalidad por malnutrición con los tratamientos apropiados, es preciso formular y aplicar pautas terapéuticas actualizadas, de carácter práctico y prescriptivo más que descriptivo, como parte de un programa integral de capacitación.

La malnutrición proteinoenergética (MPE) es un estado multicarencial que incluye una serie de trastornos cuyas formas más graves son el marasmo, el kwashiorkor y el kwashiorkor marásmico. A la labor realizada por Williams en los años treinta se le considera la primera investigación sobre MPE; dicha investigadora identificó la malnutrición edematosa en la Costa de Oro en 1933 y posteriormente, en 1935, introdujo el término "kwashiorkor".

\footnotetext{
Se publicó en inglés en el Bulletin of the World Health Organization, Vol. 74, No. 2, 1996, con el título "Why have mortality rates for severe malnutrition remained so high?" (c) Organización Mundial de la Salud, 1996.

2 Escuela de Higiene y Medicina Tropical de Londres, Londres, Inglaterra. Las solicitudes de separatas en inglés deben enviarse a A. Ashworth a la siguiente dirección postal: London School of Hygiene and Tropical Medicine, 2 Taviton Street, London WC1 OBT, Inglaterra.
}

En los años cincuenta las Naciones Unidas y sus organismos comenzaron a participar en la evaluación de la magnitud del problema y la MPE se convirtió en objeto de estudio en todo el mundo. Desde entonces se han dedicado abundantes recursos a perfeccionar el tratamiento de personas con esta afección, que es grave y complicada. No obstante, las tasas de letalidad en pacientes que reciben tratamiento fluctúan de 4,4 a $49,0 \%$ y son más altas en niños con edema.

En mayo de 1993, en una reunión a la que asistieron representantes de la OMS, del Fondo de las Naciones Unidas para la Infancia (UNICEF) y del Fondo de Ayuda a la Infancia (FAI), junto con académicos de prestigio y médicos con una amplia experiencia, se consideró qué medidas se pueden tomar, si es que alguna se presta, para combatir la letalidad persistentemente elevada que se asocia con la MPE. Se recomendó mejorar la capacitación y, con el fin de identificar áreas específicas en que se necesita adiestramiento, se juzgó aconsejable reunir más información sobre las prácticas actuales en lo referente al tratamiento de casos y revisar más a fondo los datos que revelan que la letalidad es elevada. En este trabajo se examinan estos datos y se trata de explicar por qué sigue siendo alta la mortalidad al demostrarse que hay una amplia difusión de prácticas anticuadas y deficientes en lo concerniente al tratamiento de casos de MPE.

\section{MATERIALES Y MÉTODOS}

Se recopilaron datos sobre la letalidad de la MPE mediante la revisión de las publicaciones que han aparecido en los últimos 50 años. Se obtuvieron 64 
conjuntos de datos (1-62), a los que se agregaron tres conjuntos de datos inéditos. ${ }^{3}$ A partir de noviembre de 1993 se reunió información sobre el tratamiento de casos mediante encuestas a base de dos cuestionarios enviados por correo. El primer cuestionario exploraba las prácticas actuales en hospitales y unidades de rehabilitación nutricional en todas partes del mundo y pedía información sobre el tratamiento de la MPE grave. Se enviaron los cuestionarios a las oficinas del UNICEF y del FAI en los países para su distribución local, y a los hospitales y unidades de rehabilitación conocidos por nosotros o nuestros colegas. Si la MPE grave era frecuente en una zona, se pedía al personal de salud de alto nivel que completara el cuestionario y en total se recibieron 79 contestaciones.

La segunda encuesta proporcionó pruebas anecdóticas de que hay prácticas inapropiadas. Un cuestionario sencillo, diseñado para recoger información sobre prácticas clínicas y dietéticas específicas, fue enviado a expertos que viajan mucho y observan las prácticas de tratamiento durante su trabajo. También se invitó a los profesionales incluidos en la encuesta a nombrar los países donde habían observado prácticas inapropiadas.

\section{RESULTADOS}

\section{Pruebas de la alta letalidad asociada con la MPE}

En el cuadro 1 se presentan datos sobre la letalidad en los años cincuenta a noventa que fueron reunidos en 67 hospitales y unidades de rehabilitación para el tratamiento de niños con MPE grave. En la actualidad, la mediana de la tasa de letalidad es de $23,5 \%$, por-

\footnotetext{
3 Padlan AA. A study of malnourished children in the National Children's Hospital, Quezon City, Philippines. [Tesis]. Universidad de las Indias Occidentales, Kingston, Jamaica; 1969. Mohammed MA. Evaluation of current strategies in the management of protein energy malnutrition among children under five years old, Balbala Hospital (Djibouti). [Tesis de maestría]. Escuela de Higiene y Medicina Tropical de Londres, Universidad de Londres; 1993. Pettifor J. Universidad de Witwtersrand, datos inéditos, 1993.
}

CUADRO 1. Medianas de las tasas de letalidad y recorrido de la mortalidad en niños tratados por malnutrición proteinoenergética grave, agrupados por decenio

\begin{tabular}{ccc}
\hline Década & $\begin{array}{c}\text { No. de } \\
\text { estudios }\end{array}$ & \multicolumn{2}{c}{$\begin{array}{c}\text { Mediana de } \\
\text { la letalidad }(\%)\end{array}$} \\
\hline 1950 & 7 & $20,0(9,0-50,0)^{\mathrm{a}}$ \\
1960 & 24 & $25,7(11,0-52,5)$ \\
1970 & 6 & $24,5(16,0-37,0)$ \\
1980 & 20 & $13,7(3,3-51,0)$ \\
1990 & 10 & $23,5(4,4-49,0)$ \\
\hline
\end{tabular}

Fuentes: Referencias 1 a 62 y nota 3 al pie de la página. aLas cifras entre paréntesis son los valores extremos del recorrido.

centaje más alto que el observado en los años cincuenta. De hecho, las pruebas son contundentes. No reflejan en modo alguno los conocimientos sobre la etiología y fisiopatología de la enfermedad que se adquirieron en los años cincuenta y sesenta, ni los avances más recientes. Revelan, por añadidura, que existe una tendencia generalizada a no usar tratamientos ya conocidos desde hace muchos años.

La mitad de las fuentes examinadas también proporcionaron datos desglosados por diagnóstico clínico (cuadro 2), pudiéndose observar que entre los casos de kwashiorkor y kwashiorkor marásmico era mayor la proporción de niños que fallecían. Aun en los años noventa, se notificaron tasas de letalidad de 50 a $60 \%$ en niños con malnutrición edematosa. Parece poco probable que estas tasas elevadas se vinculen con el síndrome de inmunodeficiencia adquirida (sida). Además, la emaciación es más frecuente que el edema en casos de malnutrición secundaria provocada por el sida. En el Hospital Balbala de Djibouti, ${ }^{4}$ la mortalidad fue de $12 \%$ entre los niños con infecciones respiratorias agudas o gastroenteritis, pero cuando los niños hospitalizados con estas infecciones tenían la complicación adicional de la MPE, las tasas de mortalidad se elevaron a casi $25 \%$, lo cual destaca la necesidad de mejorar el tratamiento de la MPE asociada con otros trastornos.

\footnotetext{
4 Véase la nota 3.
}

\section{Datos sobre prácticas anticuadas en los centros de tratamiento}

Las respuestas a los 79 cuestionarios revelaron el empleo de prácticas anticuadas. En particular, se solicitó información sobre el tratamiento dietético, el tratamiento inicial con antibióticos y el de la deshidratación. Actualmente se considera que el tratamiento dietético temprano debe incluir la restricción de la ingesta de proteínas y energía (63). Se recomienda un consumo diario de 1 a 2 g de proteínas y de 80 a $120 \mathrm{kcal}$, en ambos casos por $\mathrm{kg}$ de peso corporal. Las ingestas más elevadas son inadecuadas y pueden ser peligrosas en los primeros días del tratamiento porque el niño malnutrido no soporta la carga metabólica adicional. Por consiguiente, se requieren cantidades de proteínas y energía cuidadosamente controladas y administradas con frecuencia durante el día y la noche, para evitar sobrecargar el corazón, los riñones y los intestinos. Como indica el cuadro 3 en lo respectivo a las proteínas y la energía, más de $50 \%$ de las instituciones encuestadas no administraron cantidades dentro de los márgenes ideales. Esta cifra global oculta, sin embargo, las prácticas regionales, que evidentemente tendieron a ser superiores en América Latina, mientras que en África y Asia mostraron un atraso sostenido en ese respecto. Scherbaum ha llegado a la conclusión de que la alimentación con fines terapéuticos obedece a la influencia predominante del antiguo "dogma de las proteínas", es decir, la creencia en la necesidad de una dieta con un contenido alto de proteínas, sin tener en cuenta los conocimientos científicos más recientes. ${ }^{5}$

Todos los niños con malnutrición grave tienen carencias de potasio $y$ magnesio que menoscaban una serie de funciones metabólicas, entre ellas el equilibrio hidroelectrolítico. En ausencia de magnesio, se deteriora la reposi-

\footnotetext{
Scherbaum V. Management and rehabilitation of protein energy malnutrition in Ethiopia. [Tesis de maestría]. Instituto de Salud Infantil, Universidad de Londres; 1992.
} 
ción de potasio, por lo que se recomienda administrar sistemáticamente suplementos de potasio y magnesio. El cuadro 4 muestra que menos de la mitad de los centros encuestados administraban suplementos de potasio y que solo $26 \%$ administraban magnesio. El cobre y el cinc desempeñan una función importante como cofactores en la eliminación de radicales libres, con lo cual impiden la peroxidación de los lípidos y el daño a la membrana celular; no obstante, solo 14 y $25 \%$ de los centros, administraban suplementos de cobre y de cinc, respectivamente (cuadro 4). La ingesta de hierro libre en la dieta genera, por acción catalítica, radicales libres altamente reactivos; aunque no se recomienda recetar suplementos de hierro en la fase inicial del tratamiento, $31 \%$ de los centros los administraban.

Los suplementos multivitamínicos, importantes para la restauración de los tejidos afectados, se proporcionaban en $79 \%$ de los centros; 60 y $66 \%$ de estos administraban ácido fólico y vitamina $A$, respectivamente. Cuando se desglosaron por región los resultados de la administración de suplementos, se advirtió un patrón similar al de las proteínas y la energía: América Latina mostró uniformemente prácticas mejores que las observadas en Asia o en África.

Los antibióticos de amplio espectro deben administrarse siempre que se hospitalicen casos de MPE de gravedad, pero se administraban solo en $24 \%$ de los centros estudiados; las proporciones por región fueron de $4 \%$ en Asia, 32\% en África y 33\% en América Latina.

La rehidratación es, a menudo, una medida inicial necesaria en el tratamiento de la MPE. El tipo de solución de rehidratación oral es importante porque las que tienen un contenido alto de sodio pueden inducir insuficiencia cardíaca (64). Se preguntó qué soluciones de rehidratación se usaban en los centros; la recomendada por la OMS y el UNICEF, que posee un contenido alto de sodio, se empleaba en 84,74 y $56 \%$ de los centros en África, Asia y América Latina, respectivamente (cuadro 5), práctica que se
CUADRO 2. Medianas de las tasas de letalidad de la malnutrición proteinoenergética, agrupadas por diagnóstico, en los años ochenta y noventa

\begin{tabular}{lcc}
\hline \multicolumn{1}{c}{ Diagnóstico } & No. de estudios & Mediana de la letalidad (\%) \\
\hline Años ochenta & 12 & $30,1(4,3-50,0)^{\mathrm{a}}$ \\
$\quad$ Kwashiorkor & 9 & $32,3(3,7-43,4)$ \\
Kwashiorkor marásmico & 10 & $24,0(3,0-36,5)$ \\
$\quad$ Marasmo & 6 & $24,0(6,7-50,0)$ \\
Años noventa & 5 & $30,0(5,3-60,8)$ \\
$\quad$ Kwashiorkor & 6 & $19,6(6,8-35,0)$ \\
Kwashiorkor marásmico & 6 &
\end{tabular}

a Las cifras entre paréntesis son los valores extremos del recorrido.

CUADRO 3. Distribución de las instituciones por región, según sus metas en cuanto a proteínas y energía en la primera semana del tratamiento de la malnutrición proteinoenergética

\begin{tabular}{lcccc}
\hline & \multicolumn{4}{c}{ Porcentaje de las instituciones en: } \\
\cline { 2 - 5 } \multicolumn{1}{c}{ Meta } & $\begin{array}{c}\text { Todas las regiones } \\
(n=79)\end{array}$ & $\begin{array}{c}\text { Asia } \\
(n=23)\end{array}$ & $\begin{array}{c}\text { África } \\
(n=23)\end{array}$ & $\begin{array}{c}\text { América Latina } \\
(n=18)\end{array}$ \\
\hline Proteínas (g/kg/día) & 42 & 26 & 31 & 83 \\
$1-2$ & 30 & 52 & 29 & 6 \\
$3-4$ & 12 & 9 & 16 & 6 \\
$\geq 5$ & 16 & 13 & 24 & 5 \\
No especificada & & & & 17 \\
Energía (kcal/kg/día) & 9 & 13 & 29 & 61 \\
$<80$ & 35 & 26 & 29 & 17 \\
$80-119$ & 23 & 22 & 18 & 5 \\
$120-149$ & 17 & 26 & 21 & 5 \\
$150-200$ & 16 & 13 & & 5 \\
No especificada & & & & \\
\hline
\end{tabular}

CUADRO 4. Distribución de las instituciones que proporcionan $\boldsymbol{n}$ suplementos dietéticos, por región

\begin{tabular}{lcccc}
\hline & \multicolumn{4}{c}{ Porcentaje de las instituciones en: } \\
\cline { 2 - 5 } \multicolumn{1}{c}{ Suplemento } & $\begin{array}{c}\text { Todas las regiones } \\
(n=79)\end{array}$ & $\begin{array}{c}\text { Asia } \\
(n=23)\end{array}$ & $\begin{array}{c}\text { África } \\
(n=23)\end{array}$ & $\begin{array}{c}\text { América Latina } \\
(n=18)\end{array}$ \\
\hline Potasio & 48 & 35 & 47 & 61 \\
Magnesio & 26 & 22 & 18 & 44 \\
Cobre & 14 & 9 & 3 & 44 \\
Cinc & 25 & 30 & 5 & 56 \\
Multivitaminas & 79 & 74 & 74 & 89 \\
Ácido fólico & 60 & 52 & 61 & 61 \\
Vitamina A & 66 & 83 & 53 & 11 \\
Hierro: semana 1 & 31 & 30 & 39 & 67 \\
Hierro: semana 2 & 49 & 52 & 37 & \\
\hline
\end{tabular}


opone a la recomendación médica actual de emplear una solución modificada con un contenido más bajo de sodio (64). Contrario a lo recomendado (63), la utilización de la rehidratación intravenosa de manera habitual fue elevada en América Latina (22\% de los centros).

Datos anecdóticos sobre prácticas inapropiadas. Los resultados de la pequeña encuesta efectuada para recopilar información sobre el tratamiento inapropiado de los casos se presentan en el cuadro 6. Los datos fueron reunidos por 27 profesionales con experiencia en el tratamiento de la MPE grave en 34 países. Confirmando lo señalado por Scherbaum, 56\% de los expertos indicaron que el kwashiorkor se consideraba una carencia exclusivamente proteínica, siendo esta creencia la razón por la cual se recetaban dietas ricas en proteínas. De 11 a 56\% de los centros notificaron algunas prácticas, como la de no alimentar al niño ni mantenerlo abrigado en la noche, que podrían tener serias consecuencias e incluso provocar la muerte.

\section{DISCUSIÓN}

Hay pruebas muy patentes de que la mortalidad por MPE se mantiene elevada y de que hay una difusión de prácticas. Aunque en el plano mundial ha habido una disminución de la prevalencia general de MPE (65), gracias a mejoras en la atención primaria de salud y en el grado de conocimiento de la comunidad, no se ha producido un aumento concomitante de la supervivencia en pacientes con MPE grave.

Las altas tasas de mortalidad aquí presentadas, que son inadmisibles, pueden hasta representar una subestimación. Por ejemplo, en algunos hospitales, especialmente en África, los padres pueden llevarse a la casa a un niño que está a punto de morir. Tampoco se intentó obtener una muestra representativa de instituciones $y$ es probable que los centros encuestados representen una mayor proporción de los centros mejor administrados que

CUADRO 5. Distribución de los tipos de solución de rehidratación administrados, por región

\begin{tabular}{lcccc}
\hline & \multicolumn{4}{c}{ Porcentaje de las instituciones en: } \\
\cline { 2 - 5 } \multicolumn{1}{c}{ Solución } & $\begin{array}{c}\text { Todas las regiones } \\
(n=79)\end{array}$ & $\begin{array}{c}\text { Asia } \\
(n=23)\end{array}$ & $\begin{array}{c}\text { África } \\
(n=23)\end{array}$ & $\begin{array}{c}\text { América Latina } \\
(n=18)\end{array}$ \\
\hline $\begin{array}{l}\text { Intravenosa } \\
\begin{array}{l}\text { De rehidratación oral } \\
\text { de la OMS y el UNICEF }\end{array}\end{array}$ Otra $^{\text {Otra }}$ & 75 & - & - & 22 \\
\hline
\end{tabular}

de aquellos cuya administración es inferior.

Es un hecho certero que se puede lograr una baja mortalidad por MPE. De los 67 conjuntos de datos examinados, $15 \%$ mostraron tasas de mortalidad inferiores a $10 \%$, siendo la más baja de 3,3\%. Datos como estos, pese a que son pocos, revisten importancia porque demuestran que es factible reducir notablemente la mortalidad que acompaña a la MPE grave. En la Unidad de Nutrición Infantil de Dacca, en Bangladesh, la tasa de letalidad bajó de 20 a 5\% en un período de 4 años (66) sin que hubiera ningún cambio en la gravedad de los pacientes en el momento de la hospitalización. La reducción de la mortalidad se atribuyó a las siguientes modificaciones en el tratamiento de los casos: la prescripción de antibióticos de amplio espectro al hospitalizar a todo paciente; la transfusión de concentrados celulares en los casos de anemia grave; la omisión de suplementos de hierro en la primera semana de tratamiento; la práctica de no dar rehidratación intravenosa siempre que sea posible; el reinicio cauteloso de la alimentación; el empleo de una dieta con un bajo contenido de sodio; y la vigilancia diaria para detectar signos de sobrecarga de líquidos.

El examen de los distintos regímenes aplicados en el mundo reveló que en América Latina las prácticas eran, en general, de mejor calidad. Los puntos iniciales para la distribución de los cuestionarios en América Latina fueron, entre otros, unidades de rehabilitación reconocidas en el ámbito internacional por sus exitosas investigaciones sobre el tratamiento de la MPE. Puede ser, por consiguiente, que la diferencia regional observada en el caso de América Latina se haya originado en un sesgo muestral.

Hay pruebas, sin embargo, de que en África el mayor empobrecimiento de
CUADRO 6. Distribución de las prácticas inapropiadas que refirieron los médicos expertos

\begin{tabular}{lc}
\hline \multicolumn{1}{c}{ Práctica inapropiada } & $\begin{array}{c}\text { Porcentaje de los médicos } \\
(n=27)\end{array}$ \\
\hline Se administran diuréticos para tratar el edema & 41 \\
No se reconoce la necesidad de un tratamiento específico para la & \\
"fase aguda" & 41 \\
El kwashiorkor se considera una carencia proteínica; se administra de & 56 \\
inmediato una dieta rica en proteínas & 37 \\
No siempre se administran dosis altas de vitamina A, aunque exista la & 56 \\
carencia & 11 \\
Se trata la anemia con hierro desde que el paciente ingresa & 61 \\
Se administran albúmina y aminoácidos por vía intravenosa & 41 \\
No se mide la cantidad de alimentos & 33 \\
No se da alimentación por la noche & \\
No se usan colchas, aunque se corra el riesgo de hipotermia & \\
\hline
\end{tabular}


los hospitales impide que se proporcionen suministros médicos y servicios clínicos esenciales, y ello podría limitar las prácticas adecuadas (67, y A. Burgess, comunicación personal, 1995).

Si se dejan a un lado estas reservas, es posible que una causa importante de la elevada tasa de letalidad por MPE radique en prácticas defectuosas porque los conocimientos son inadecuados; entonces resulta conveniente perfeccionar los métodos usados para capacitar a los profesionales que ejercen la medicina y mejorar la información que se les transmite. Todas las pautas ampliamente disponibles han sido generadas por la OMS $(68,69)$ y por organizaciones no gubernamentales como el Oxfam (70) y el FAI (71). Las pautas difieren en su claridad, su fundamentación en los conocimientos más recientes y sus instrucciones terapéuticas. Se carece de un conjunto definitivo de pautas prácticas y fáciles de aplicar. Pese a que su ausencia no se puede tomar como causa directa de la letalidad elevada, es factible que con- tribuya al problema. Urge contar con información clara, accesible y autorizada, de carácter más prescriptivo que descriptivo, para facilitar la toma de decisiones rápidas y acertadas.

Los conocimientos de los profesionales médicos y paramédicos se podrían incrementar mediante cursos de capacitación a corto plazo y, en el plazo más largo, mediante la actualización y modificación de los cursos y planes de estudio en las instituciones de educación terciaria. Paralelamente, las instituciones sanitarias podrían revisar sus propios tratamientos clínicos y su desempeño con vistas a mejorar la práctica. El proceso ha de comenzar con la preparación de materiales educativos y de pautas sencillas y claras basadas en la experiencia adquirida con el uso de tratamientos existentes de comprobada eficacia. La OMS y el UNICEF están contribuyendo a este proceso mediante su programa denominado Tratamiento Integrado del Niño Enfermo, en el que se establecen criterios para el envío de casos a otros servicios, pautas técnicas y materiales de capacitación para el tratamiento de las enfermedades infantiles más comunes (diarrea, infecciones respiratorias agudas, sarampión, malaria y malnutrición). Con un mejor tratamiento de los casos se podrían evitar aproximadamente 0,5 millones de defunciones anuales vinculadas con la malnutrición; por consiguiente, urge que se produzcan adelantos en este aspecto.

Agradecimiento. Agradecemos en especial las contribuciones de A. Jackson, D. J. Millward y S. Khanum, así como la de todas aquellas personas y miembros de las oficinas del UNICEF en los países que respondieron a los cuestionarios.

Este trabajo fue patrocinado por el UNICEF y por la Agencia Canadiense para el Desarrollo Internacional (CIDA).

\section{REFERENCIAS}

1. Achar ST. Nutritional dystrophy among children in Madras. Br Med J 1950;1:701-703.

2. Brown $\mathrm{KH}$, et al. Infections associated with severe protein-calorie malnutrition in hospitalised infants and children. Nutr Res 1981; 1:33-46.

3. Chaudhuri RN, et al. Kwashiorkor and marasmus in Calcutta. J Indian Med Assoc 1961;36:557-565.

4. Christie DC, et al. Coagulase-negative staphylococcal bacteremia in severely malnourished Jamaican children. Ped Infect Dis J 1992; 11:1030-1036.

5. Cook R. Is hospital the place for the treatment of malnourished children? Environ Chil Health 1971;17:1-25.

6. Cooper E, et al. Caribbean children, thriving and failing, in and out of hospital. J Trop Pediatr 1980;26:232-238.

7. Coulter JBS, et al. Protein-energy malnutrition in northern Sudan: clinical studies. Eur J Clin Nutr 1988;42:787-796.

8. Cousens S, et al. Prolonged breast-feeding: no association with increased risk of clinical malnutrition in younger children in Burkina Faso. Bull World Health Organ 1993;71:713-722.

9. De Silva CC. Common nutritional disorders of childhood in the tropics. En: Advances in pediatrics, vol. XIII. Chicago: Year Book Medical Publishers; 1964:213-164.

10. Erinoso $\mathrm{HO}$, et al. Prognostic factors in severely malnourished hospitalised Nigerian children. Trop Geogr Med 1993;45:290-293.

11. Friedland IR. Bacteraemia in severly malnourished children. Ann Trop Pediatr 1992;12: 433-440.

12. Garrow JS, et al. The treatment and prognosis of infantile malnutrition in Jamaican children. West Indian Med J 1962;11:217-226.

13. Garrow JS, Pike MC. The short-term prognosis of severe infantile malnutrition. Br J Nutr 1967;21:155-165.

14. Gelfand M, Carr WR. The results of treatment of kwashiorkor in Salisbury, with high protein diets. Cent Afr J Med 1956;2:425-427.

15. Gillam SV. Mortality risk factors in acute protein-energy malnutrition. Trop Doct 1989;19: 82-85.

16. Glatthaar LL, et al. Protein-energy malnutrition: the role of nutrition education in rehabilitation. Hum Nutr Clin Nutr 1985;40C: 271-285.

17. Gottleib A, Kochman R. [Hipoalbuminemia, edema y su tratamiento en 50 casos de malnutrición infantil]. Harefuah 1957;52:193-197 (en hebreo, con resumen en inglés).
18. Gomez F, et al. Mortality in second- and thirddegree malnutrition. J Trop Pediatr 1956;2: 77-83.

19. Hedayat H. Protein-calorie malnutrition in hospitalised Iranian children. J Trop Pediatr 1968;14:124-131.

20. Hone NM, Fermor JK. High energy feeding for protein-calorie malnutrition. Trop Doct 1987;17:179-181.

21. Husaini YK, et al. Outpatient rehabilitation of severe protein-energy malnutrition (PEM). Food Nutr Bull 1982;8:55-59.

22. Ibekwe VE, Ashworth A. Management of protein-energy malnutrition in Nigeria: an evaluation of the regimen at Kersey Nutrition Rehabilitation Centre. Trans Roy Soc Trop Med Hyg 1994;88:594-595.

23. Kahn E. Prognostic criteria of severe protein malnutrition. Am J Clin Nutr 1959;7:161-165.

24. Kahn AA. A review of the year's paediatric admissions to the Kenyatta National Hospital, Nairobi, Kenya. J Trop Med Hyg 1966;69:7-11.

25. Keet MP, et al. Kwashiorkor: a prospective ten year follow-up study. South Afr Med J 1971; 45:1427-1434.

26. Khanum S, Kabir I. Survival and growth of severe protein-energy malnutrition children 2 years after nutrition rehabilitation in an urban 
nutrition centre in Bagladesh. J Trop Pediatr 1989;30:138-139.

27. Khanum S, et al. Severe protein-energy malnutrition in urban Dhaka, and their response to treatment. Bangladesh J Nutr 1987;1:1-9.

28. Khin-Maung $U$, et al. Beyond rehydration therapy: nondehydration deaths from diarrhoea in children with malnutrition. $J$ Trop Pediatr 1992;38:165-267.

29. Laditan AAO, Tindimebwa G. The proteinenergy malnourished child in a Nigerian teaching hospital. J Trop Pediatr 1983;29:61-64.

30. Lamptey PR, et al. Comparison of nutriwards and nutrihuts in the northern Mindanao region, Republic of Philippines. Food Nutr Bull 1980;3:8-17.

31. Lawless J, Lawless MM. Kwashiorkor: a result of cold injury in a malnourished child. Lancet 1963;1:972-974.

32. Marcande E, et al. La mala nutrición en el primer año de vida: terminología y observaciones clínicas basadas en la revisión de 230 casos de kwashiorkor y 41 casos de marasmo. Rev Hosp Clin Fac Med Sao Paulo 1962;18:17-38 (en portugués).

33. McFie J, Yarom R. Child health in Luluabourg, Congo (with a note on the age distribution of kwashiorkor). J Trop Pediatr 1962;7:123-128.

34. McLaren DS, et al. The socio-economic background of marasmus in Lebanon. Leban Med J 1964;17:85-96.

35. Mitra AK, et al. Complications and outcome of disease in patients admitted to the intensive care unit of a diarrhoeal diseases hospital in Bangladesh. Trans Roy Soc Med Hyg 1991; 85:685-687.

36. Musoke LK. An analysis of admissions to the paediatric division, Mulago hospital in 1959. Arch Dis Child 1961;36:305-306.

37. Neave M. Protein calorie malnutrition of early childhood in Western Samoa. Trop Geogr Med 1968;20:191-201.

38. Netrasiri A, Netrasiri C. Kwashiorkor in Bangkok (analytical study of 54 cases). J Trop Pediatr 1955;7:425-431.

39. Ogbeide MI. The influence of protein-calorie malnutrition on the pattern of pre-school childhood diseases. Clin pediatr 1968;7: 425-431.

40. Ojofeitimi EO, Teniola SO. Evaluation of nutrition re-habilitation centre in Ife-Ife, Oyo State, Nigeria. Word Rev Nutr Diet 1980;35:87-95.
41. Omer HO, et al. Pattern of protein-energy malnutrition in Sudanese children and comparison with some other Middle East countries. I Trop Pediatr 1975;21:329-333.

42. Pecoul B, et al. Efficiency of a therapeutic feeding centre evaluated during hospitalization and a follow-up period, Tahoka, Niger 1987-1988. Ann Trop Pediatr 1992;12:27-54.

43. Pereira SU, Begum A. The manifestation and management of severe protein-calorie malnutrition (kwashiorkor). World Rev Nutr Diet 1974;19:1-50.

44. Pharaon HM. Protein-calorie malnutrition of early childhood in Jordan. J Trop Pediatr 1962; 8:53.

45. Pharaon HM, Hijazi SS. A review of proteincalorie malnutrition of early childhood in Jordan. Jordan Med J 1967;2:305.

46. Reeds PH, Laditan AAO. Serum albumin and transferrin in protein-energy malnutrition: their use in the assessment of marginal undernutrition and the prognosis of severe undernutrition. Br J Nutr 1976;36:255-263.

47. Ramdath DD, Golden MHN. Elevated glutathione $S$-transferase activity in erythrocytes from malnourished children. Eur J Clin Nutr 1993;47:658-665.

48. Ramos-Galvan R, Calderon JM. Deaths among children with third-degree malnutrition. Am J Clin Nutr 1965;16:351-263.

49. Ramprasad V, Williams Van W. Education through nutrition rehabilitation. J Trop Pediatr 1980;26:123-126.

50. Sadre M, et al. Dehydration syndrome in malnourished Iranian children. Am J Clin Nutr 1969;22:139-146.

51. Sadre M, Donoso G. Treatment of malnutrition. Lancet 1969;1759-1766.

52. Scragg J, Rudbridge C. Kwashiorkor in African children in Durban. Br Med J 1960; 11:1759-1766.

53. Sinha DP. Hospital admission for malnutrition trends in the English-speaking Caribbean. Cajanus 1987;20:179-190.

54. Smith IF, et al. Plant protein rehabilitation diets and iron supplementation of the proteinenergy malnourished child. Eur I Clin Nutr 1989;43:763-768.

55. Tolboom JJM, et al. Severe protein-energy malnutrition in Lesotho: death and survival in hospital; clinical findings. Trop Geogr Med 1986;38:351-358.
56. Van Roosmalen-Wiebenga MW, et al. Nutrition in a rehabilitation hospital-a waste of time and money? Evaluation of nutritional rehabilitation in a rural district hospital in South-West Tanzania 11. Long term results. J Trop Pediatr 1987;33:24-28.

57. Venkatachalem PS. Clinical features of nutritional oedema syndrome in children. Indian J Med Res 1954;42:555-568

58. Vis HL. On the treatment of certain forms of protein-energy malnutrition in childhood with respect to fatal complications (an example from rural Central Africa). Ann Nestle 1985; 43:19-30.

59. Wayburne S. Malnutrition in Johannesburg. En: McCance RA, Widdowson EM, eds. Calorie deficiencies and protein deficiencies. London: Churchill; 1986:7-20.

60. Wharton BA. The mortality of malnutrition in early childhood. Environ Child Health 1971;13: 17-24.

61. Yarom R, McFie J. Kwashiorkor in the Congo (a clinical survey of successive cases in Kasai Province). J Trop Pediatr 1963;9:56-63.

62. Zaki LA. Kwashiorkor in Iraq. J Faculty Med (Baghdad) 1968;10:64.

63. Waterlow JC. Protein-energy malnutrition. London: Arnold; 1992.

64. Briend A, Golden MHN. Treatment of severe child malnutrition in refugee camps. Eur J Clin Nutr 1993;47:750-754.

65. United Nations Children's Fund. Child malnutrition: progress towards the World Summit for Children goal. New York: UNICEF; 1993.

66. Khanum S, et al. Controlled trial of three approaches to the treatment of severe malnutrition. Lancet 1994;344:1728-1732.

67. Brewster D, Manary M. Treatment of severe malnutrition. Lancet 1995;345:453.

68. World Health Organization. The treatment and management of severe protein-energy malnutrition. Geneva: WHO; 1981.

69. World Health Organization. The management of nutritional emergencies in large populations. Geneva: WHO; 1978

70. Oxfam. Oxfam's practical guide to selective feeding programmes Oxford: Oxfam; 1978.

71. Appleton J. Drought relief in Ethiopia, a practical guide. London: Save the Children; 1987.
ABSTRACT

\section{Why have mortality rates for severe malnutrition remained so high?}

A review of the literature that has appeared over the past five decades indicates that the median case fatality from severe malnutrition has remained unchanged over this period and is typically $20-30 \%$, with the highest levels (50-60\%) being among those with edematous malnutrition.

A likely cause of this continuing high mortality is faulty case-management. A survey of treatment centres worldwide $(n=79)$ showed that for acutely ill children, inappropriate diets that are high in protein, energy and sodium and low in micronutrients are commonplace. Practices that could have fatal consequences, such as prescribing diuretics for edema, were found to be widespread. Evidence of outmoded and conflicting teaching manuals also emerged. Since low mortality levels from malnutrition can be achieved using appropriate treatment regimens, updated treatment guidelines, which are practical and prescriptive rather than descriptive, need to be implemented as part of a comprehensive training programme. 